\title{
The application and value of continuous nursing in patients after coronary artery bypass grafting
}

\author{
Sheng-Huo Zhou $^{1 \dagger}$, Shu-Ting Huang ${ }^{1 \dagger}$, Ning $\mathrm{Xu}^{1 \dagger}$, Qiang Chen ${ }^{1 *}$ (D), Liang-Wang Chen ${ }^{1}$ and Yur-Ren Kuo ${ }^{2}$
}

\begin{abstract}
Objective: To investigate the application and value of continuous nursing after coronary artery bypass grafting.

Methods: The clinical data of 62 patients after coronary artery bypass grafting from January 2016 to January 2018 were analyzed retrospectively. According to the nursing mode, the patients were divided into two groups: the continuous nursing group $(n=30)$ and the conventional nursing group $(n=32)$. All patients completed Self-Rating Depression Scale (SDS) and Self-Rating Anxiety Scale (SAS) at admission and 1 year after operation. All patients completed Seattle Angina Pectoris Questionnaire (SAQ) at discharge and 1 year after operation.

Results: All patients were followed up for more than one year. One year after operation, SAQ score in five items in continuous nursing group was significantly better than that in conventional nursing group. $(P<0.05)$ The continuous nursing group exhibited significantly decreased SAS and SDS scores 1 year after surgery compared to the preoperative SAS and SDS scores. $(P<0.05)$ The SAS and SDS scores of the continuous nursing group were significantly better than those of the conventional nursing group 1 year after surgery. $(P<0.05)$ Then incidence rate of chest tightness or chest pain and coronary restenosis in continuous nursing group were significantly less than that in conventional nursing group. $(P<0.05)$.

Conclusion: Continuous nursing improved patient compliance with treatment and reduces the occurrence of complications. The patient also receives proper psychological evaluations, which relieve patient anxiety and depression and improve the quality of life.
\end{abstract}

Keywords: Continuous nursing, coronary artery bypass grafting, Quality of life

\section{Introduction}

Coronary artery disease is a kind of heart disease of myocardial ischaemia, hypoxia or myocardial necrosis caused by organic stenosis or obstruction of the coronary artery, and it is one of the diseases with the highest mortality rates in the world $[1,2]$. Coronary artery bypass grafting is an effective method to treat such

\footnotetext{
* Correspondence: chenqiang2228@163.com

†'Sheng-Huo Zhou, Shu-Ting Huang and Ning Xu contributed equally to this work.

'Department of Cardiovascular Surgery, Union Hospital, Fujian Medical University, Fuzhou 350001, People's Republic of China

Full list of author information is available at the end of the article
}

disease, which can effectively improve the symptoms of myocardial ischaemia [3]. But it is still with a long course and a repeated condition that requires long-term treatment, and needs long-term and repeated treatment after discharge [4]. Patients' self-management abilities and compliance with treatment decreases may be results in coronary artery restenosis that causes a heavy burden to the family and to society and even endangers the life of patients $[5,6]$. Therefore, it is very important to extend high-quality medical service to the patients and their family to understand the compliance behaviour, treatment effect and psychological state of the patients after

C C The Author(s). 2020 Open Access This article is licensed under a Creative Commons Attribution 4.0 International License, which permits use, sharing, adaptation, distribution and reproduction in any medium or format, as long as you give appropriate credit to the original author(s) and the source, provide a link to the Creative Commons licence, and indicate if changes were made. The images or other third party material in this article are included in the article's Creative Commons licence, unless indicated otherwise in a credit line to the material. If material is not included in the article's Creative Commons licence and your intended use is not permitted by statutory regulation or exceeds the permitted use, you will need to obtain permission directly from the copyright holder. To view a copy of this licence, visit http://creativecommons.org/licenses/by/4.0/ The Creative Commons Public Domain Dedication waiver (http://creativecommons.org/publicdomain/zero/1.0/) applies to the data made available in this article, unless otherwise stated in a credit line to the data. 
discharge and provide the patients with medical and psychological guidance at the same time [7-10]. The aim of this study is to explore a better nursing method for patients who have undergone coronary artery bypass grafting by summarizing the experience of continuous nursing in patients after coronary artery bypass grafting at our hospital.

\section{Materials and methods}

The present study was approved by the ethics committee of Fujian Medical University, China and adhered to the tenets of the Declaration of Helsinki. Additionally, all patients signed the consent form before participating in the study.

\section{Patients}

The clinical data of 62 patients after coronary artery bypass grafting from January 2016 to January 2018 were analyzed retrospectively. According to the nursing mode, the patients were divided into two groups: the continuous nursing group (group $\mathrm{A}, n=30$ ) and the conventional nursing group (group $\mathrm{B}, n=32$ ). All patients completed the Self-Rating Depression Scale (SDS) and Self-Rating Anxiety Scale (SAS) at admission and 1 year after surgery. All patients completed the Seattle Angina Pectoris Questionnaire (SAQ) at discharge and 1 year after operation. All the patients' general clinical data are shown in Table 1. Patients met the inclusion criteria if they underwent coronary artery bypass grafting. The following exclusion criteria were used: 1) lack of independent reading ability, 2) lack of communication equipment and difficult communication information, 3) postoperative death, 4) severe disease of other organs or 5) refusal of continuing nursing services and participation in the study.

Table 1 Comparison of general data between the two groups

\begin{tabular}{llll}
\hline Item & Group A & Group B & $P$ value \\
\hline Number & 30 & 32 & \\
Age (year) & $52.6 \pm 8.3$ & $55.6 \pm 10.1$ & 0.768 \\
Man/woman & $19 / 11$ & $21 / 11$ & 0.851 \\
Hypertension & 14 & 16 & 0.793 \\
Diabetes mellitus & 9 & 7 & 0.465 \\
Hyperlipemia & 5 & 6 & 0.830 \\
Smoking & 8 & 10 & 0.691 \\
Alcoholism & 4 & 4 & 0.922 \\
Record of formal schooling & & & \\
$\quad$ Junior high school and below & 22 & 23 & 0.782 \\
$\quad$ Senior middle school & 5 & 7 & \\
$\quad$ Universities and above & 3 & 2 & \\
$\quad$ Place of residence: rural / urban & $21 / 9$ & $22 / 10$ & 0.915 \\
\hline
\end{tabular}

\section{Nursing methods}

\section{Continuing nursing}

On the basis of conventional nursing, the method of continuous nursing involved issuing contact cards at discharge. The information on the contact card included basic patient information and the telephone number and WeChat of the attending doctor, attending nurse and head of the department. The nurse instructed the patient to join the WeChat group and provided the patient with instructions on how to use the WeChat function correctly and skilfully after surgery. One team member participated in the online WeChat support group every day from 18:00 to $21: 00 \mathrm{~h}$ to answer patients' questions, remind and monitor the patient's use of drugs and provide regular reviews, and encourage group members to actively communicate and share nursing experiences associated with the care of patients. For patients and familied with pessimism, anxiety or depression mood, we added WeChat alone to carried out psychological counselling and psychological support one-on-one via text, voice or video way.

\section{Conventional nursing}

Routine medical services were provided to patients during hospitalization. The attending physician explained disease-related knowledge and surgical procedures to patients and their families. Nursing staff provided dietary knowledge, postoperative attention, methods, matters required after discharge, regular visitation and regular reexamination of coronary angiography.

\section{Research tools}

1. SAQ: We used the SAQ with a total of 19 entries in 5 major items to evaluate the quality of life of patients [11]. The 5 items are physical activity restriction, stable angina pectoris, frequency of angina pectoris attack, satisfaction with treatment and cognitive degree of disease. Each dimension adopts the percentile system, and the higher the score, the better the quality of life.

2. SDS: Zung's SDS was applied, which is widely used in the clinic and has high reliability and validity [12]. This scale consists of 20 items, including 10 negative symptoms and 10 positive symptoms. Each question represents a feature of depression. All items together reflect the mood, body discomfort symptoms, spiritual movement, behaviour, and psychological symptoms of patients with depression. The score involves 4 grades. The scores were obtained using the scoring method in ascending order (1 to 4 ) based on the occurrence frequency of the positive symptoms. The rough scores were obtained using the reverse scoring method in descending order (4 to 1 ) based on the occurrence frequency of the negative symptoms. The standard score was obtained by multiplying the scores by 1.25 and rounding the result. 
Normally, the upper limit score is 41 , and the standard total score is 53. A higher score indicates a more significant depression tendency.

3. SAS: Zung's SAS was applied, which is widely used in the clinic and has high reliability and validity [13]. Fifteen items are stated with negative words. The scores were obtained using the scoring method in ascending order (1 to 4) based on the occurrence frequency of the symptoms. Five items are stated with positive words. The scores were obtained using the reverse scoring method in descending order (4 to 1 ) based on the occurrence frequency of the symptoms. The total score was obtained by adding the scores of all items. The standard score was obtained by multiplying the total score by 1.25 and rounding the result. The mean value of the standard score is 50 . Grade description: < 50 means normal, 50 to 59 means mild anxiety, 60 to 69 means medium anxiety, and $\geq 70$ means severe anxiety.

\section{Statistical analysis}

According to the incidence of post-discharge complications in the pre-survey, with $\alpha=0.05$, two-tailed and a power of $90 \%$, the sample sizes of the two groups were 30 and 32, respectively, calculated by SPSS18.0. Quantitative data were expressed as the mean \pm standard deviation $(\mathrm{x} \pm$ s). The continuous data were all in accordance and normally distributed by the normal test and were statistically analyzed by the independent sample $t$ test. Qualitative data were compared by chi-square test and Fisher's test. A $p$ value of $<0.05$ was defined as statistically significant.

\section{Results}

There were no significant differences between the two groups in general clinical data. There were also no significant differences in the scores of SAS and SDS at admission and SAQ at discharge between the two groups. $(P>0.05)$ There was no significant difference in the SAQ score between the two groups at discharge in 5 items: physical activity restriction, stable angina pectoris, frequency of angina pectoris attack, satisfaction with treatment and cognitive degree of disease. $(P>0.05)$ These results indicated that the two groups were homogeneous and comparable.

All patients were followed up for more than 1 year. One year after the operation, the SAQ score in the five items in the continuous nursing group was significantly better than that in the conventional nursing group. $(P<$ 0.05) (Table 2, Table 3) The SAS and SDS scores of the continuous nursing group were significantly decreased 1 year after surgery compared to the preoperative SAS and SDS scores, and the differences were statistically significant. $(P<0.05)$ However, the SAS and SDS scores of the conventional nursing group were also lower 1 year after surgery than the scores before surgery, but these
Table 2 Comparison of SAQ scores between the two groups at discharge

\begin{tabular}{llll}
\hline Item & Group A & Group B & $P$ value \\
\hline Physical activity restriction & $62.5 \pm 8.9$ & $64.2 \pm 10.4$ & 0.874 \\
Stable angina pectoris & $54.6 \pm 7.3$ & $52.5 \pm 9.6$ & 0.912 \\
Frequency of angina pectoris attack & $61.3 \pm 9.3$ & $60.3 \pm 11.2$ & 0.769 \\
Satisfaction with treatment & $73.5 \pm 10.9$ & $75.8 \pm 12.4$ & 0.816 \\
Cognitive degree of disease & $63.8 \pm 11.3$ & $66.1 \pm 13.2$ & 0.743 \\
\hline
\end{tabular}

differences were not statistically significant. $(P>0.05)$ The SAS and SDS scores of the continuous nursing group were significantly lower than those of the conventional nursing group 1 year after surgery. $(P<0.05)$ (Table 4$)$.

There were 2 patients with chest tightness or chest pain after discharge in the continuous nursing group and 8 patients in the conventional nursing group. There was a significant difference between the two groups. $(P=0.039)$ There were no patients with coronary restenosis after discharge in the continuous nursing group and 4 patients in the conventional nursing group. There was a significant difference between the two groups. $(P=0.045)$.

\section{Discussion}

With the improvement of socioeconomic level, the enrichment of material objects and the arrival of an era of ageing, the incidence of coronary artery disease is increasing in recent year [14]. coronary artery bypass grafting is one of the most effective methods for treating coronary artery disease, and patients need to take longterm anti-platelet aggregation, lipid-regulating and $\beta$ receptor blocking agents to prevent the occurrence of cardiovascular adverse events. Patients often have a history of chronic diseases such as hypertension, diabetes and hyperlipidaemia and poor habits such as smoking and drinking, and the occurrence of coronary artery disease is closely related to the aforementioned chronic diseases and habits $[15,16]$.

Most patients underwent coronary artery bypass grafting in China are from rural areas, and their academic qualifications are generally low. The lack of diseaserelated knowledge, poor self-management ability, and the lack of awareness of the importance of continuous treatment result in an inability to take drugs correctly

Table 3 Comparison of SAQ scores between the two groups 1 year after operation

\begin{tabular}{llll}
\hline Item & Group A & Group B & $P$ value \\
\hline Physical activity restriction & $83.3 \pm 13.9$ & $73.4 \pm 12.5$ & 0.032 \\
Stable angina pectoris & $77.6 \pm 9.7$ & $63.5 \pm 8.2$ & 0.024 \\
Frequency of angina pectoris attack & $80.3 \pm 11.2$ & $69.8 \pm 10.5$ & 0.018 \\
Satisfaction with treatment & $94.8 \pm 12.3$ & $80.3 \pm 11.6$ & 0.011 \\
Cognitive degree of disease & $89.5 \pm 10.9$ & $76.1 \pm 12.4$ & 0.015 \\
\hline
\end{tabular}


Table 4 Comparison of psychological state before and after nursing intervention

\begin{tabular}{|c|c|c|c|}
\hline Item & Group A & Group B & $P$ value \\
\hline \multicolumn{4}{|l|}{ The SDS score } \\
\hline Preoperation & $53.7 \pm 10.8$ & $54.3 \pm 11.7$ & 0.886 \\
\hline 1 year after operation & $41.3 \pm 9.1^{*}$ & $49.2 \pm 12.1$ & 0.031 \\
\hline \multicolumn{4}{|l|}{ The SAS score } \\
\hline Preoperation & $62.3 \pm 13.3$ & $60.2 \pm 15.6$ & 0.891 \\
\hline 1 year after operation & $46.2 \pm 10.4^{*}$ & $53.8 \pm 12.2$ & 0.028 \\
\hline
\end{tabular}

and maintain good lifestyle habits after discharge, which easily causes poor control of blood pressure, blood sugar and blood lipids, leading to adverse cardiovascular events, and even endangering the lives of patients. Some studies have shown that a deterioration of most patients' condition after coronary artery bypass grafting is not only related to the physique of the patients themselves but also to the low compliance of drug use and the lack of postoperative nursing knowledge, ways to solve the confusion, timely medical treatment and attention to the disease [17]. After coronary artery bypass grafting, most patients die from a lack of knowledge and unhealthy lifestyle, not from the disease itself [18]. Therefore, it is essential to strengthen the patients' management of coronary artery bypass grafting from becoming disconnected from hospitals, thus leaving the family to solve the problem of insufficient support after discharge [19]. As a result, a new nursing service model (continuous nursing) that extends treatment and rehabilitation care during hospitalization to the family to help improve the self-management of patients and ensure that information, treatment and nursing services continue without sudden interruption has been gradually applied to the clinic [20].

In this study, we found that the patients with chest tightness or chest pain and coronary artery restenosis in the continuous nursing group were significantly lower than those in the conventional nursing group 1 year after the operation. The continuous nursing group was significantly better than the conventional nursing group in terms of physical activity restriction, stable angina pectoris, frequency of angina pectoris attack, satisfaction with treatment and cognitive degree of disease. The results are suggested that continuous nursing can reduce the incidence of chest tightness or chest pain and coronary artery restenosis and improve the quality of life in patients after coronary artery bypass grafting.

For most people, surgery is a terrible shock, and there is tension and anxiety. Especially for the heart surgery, which has a high risk, the fear and tension are inevitable before and after surgery [21]. Patients underwent coronary artery bypass grafting requires taking lifelong drugs, strictly controlling blood pressure, blood glucose and blood lipids, and maintaining good lifestyle habits. For most patients, there will be a heavy psychological burden, and some patients even lose confidence and have obvious negative feelings [22, 23]. In addition, for many of the patients from rural areas, the degree of education is generally low and the knowledge about the disease is insufficient. In the short time during hospitalization, patients cannot fully learn all of the knowledge for the management of the disease from the nursing staff. The medical treatment in rural areas is inconvenient, and the problem is difficult to solve in a timely manner. Therefore, patients are more likely to be anxious and negative.

The sudden loss of nursing and professional knowledge would have a significant negative impact on the health and quality of life for the patients and families who have neither psychological adaptability nor learning how to care. Therefore, patients need more follow-up and attention in physical care and mental health care. WeChat with visibility functions is widely and conveniently used in China and it is available in a timely manner. We use WeChat as the main medium to carry out continuous nursing. The nurse instructed the patient to join the WeChat group and trained the patient on the use of the WeChat function correctly and skilfully after surgery. To increase patients' knowledge of the disease, strengthen their self-management ability, improve their compliance, and reduce their anxiety caused by the disease, one team member was on duty every day to answer the patients' questions, remind and monitor the patients' on the use of drugs with regular review, and encourage group members to actively communicate and share nursing experiences associated with the care of patients. For patients with severe negative feelings and anxiety, we could also communicate as soon as possible through the WeChat platform to solve the patients' problems in a timely manner and carry out special psychological counselling and support. At the same time, we were able to encourage patients to communicate with each other and share experiences to enhance confidence, ease negative psychological feelings and eliminate anxiety via the continuous nursing group of WeChat. In this study, the SAS and SDS scores of the continuous nursing group were significantly decreased 1 year after surgery compared to the preoperative SAS and SDS scores, and the SAS and SDS scores of the continuous nursing group were also significantly lower than those of the conventional nursing group1 year after surgery. These results suggest that continuous nursing for patients undergoing coronary artery bypass grafting can significantly alleviate depression and anxiety.

There are several limitations in this study. First, this study was a retrospective study with a small sample size, and patients who did not have independent reading 
ability, communication equipment or convenient information communication were not included in this study, which may cause selection bias. Second, this study was a single-centre study, and more research from multiple centres is mandatory to assess the value of continuous nursing in the future. Third, the follow-up period of this study was brief, and a longer term follow-up period is needed.

\section{Conclusion}

Continuous nursing extends high-quality hospital nursing service and psychological support to the patient's family, which improved patient compliance with treatment, reduced the occurrence of postoperative complications, and provided the patient with proper psychological evaluation to relieve anxiety and depression and improve their quality of life.

\section{Abbreviations}

SDS: Self-Rating Depression Scale; SAS: Self-Rating Anxiety Scale; SAQ: Seattle Angina Pectoris Questionnaire

\section{Acknowledgements}

We highly acknowledge the contribution by the participating doctors: Yanjuan Lin, Dao-Zhong Chen, Feng Lin, Qi-min Wang, Zhong-yao Huang, Hanfan Qiu, Xiao-fu Dai, Xue-Shan Huang, Hui Zhang, Zeng-chun Wang. Also, we wish to extend our gratitude to Xiu-jun Wang and her colleagues.

\section{Authors' contributions}

Conceived and designed the experiments: QC S-hZ. Performed the experiments: S-hZ, S-tH. Analyzed the data: NX. Contributed reagents/materials/analysis tools: QC S-hZ. Wrote the paper: S-hZ. Read and approved the final manuscript: Z-wL, StH NX QC L-wC Y-rK

\section{Funding}

This research was sponsored by Chinese national and Fujian provincial key clinical specialty construction programs.

\section{Availability of data and materials}

Data sharing not applicable to this article as no data sets were generated or analyzed during the current study.

\section{Ethics approval and consent to participate}

The present study was approved by the ethics committee of Fujian Medica University, China and adhered to the tenets of the Declaration of Helsinki.

\section{Consent for publication}

Not applicable.

\section{Competing interests}

All authors declare that they have no competing interests.

\section{Author details}

${ }^{1}$ Department of Cardiovascular Surgery, Union Hospital, Fujian Medical University, Fuzhou 350001, People's Republic of China. ${ }^{2}$ Division of Plastic Surgery, Department of Surgery, Kaohsiung Medical University Hospital, 100 TzYou 1st Rd, Kaohsiung City 80756, Taiwan.

Received: 29 January 2020 Accepted: 25 June 2020

Published online: 10 July 2020

\section{References}

1. Goch A, Misiewicz P, Rysz J, Banach M. The clinical manifestation of myocardial infarction in elderly patients. Clin Cardiol. 2009;32(6):E46-51.

2. Rosamond W, Flegal K, Furie K, Go A, Greenlund K, Haase N, et al. American Heart Association Statistics Committee and StrokeStatistics Subcommittee
Heart disease and stroke statistics 2008 update:a report from the American Heart Association tatistics committee and stroke statistics subcommittee. Circulation. 2008;117(4):e25-146.

3. Lawie GM, Morris GC, Earle IV. Long-term results of coronary artery bypass grafting, analysis of 1698 patients followed 15 to 20 years. Ann Surg. 1991; 213(5):377-8.

4. Henderson A, Zernike W. A study of the impact of discharge information for surgical patients. J Adv Nurs. 2001;35(3):435-41.

5. Hammill BG, Curtis LH, Schulman KA, Whellan DJ. Relationship between cardiac rehabilitation and long-term risks of death and myocardial infarction among elderly Medicare beneficiaries. Circulation. 2010;121(1):63-70.

6. Scholte op Reimer WJ, Jansen $\mathrm{CH}$, de Swart EA, Boersma E, Simoons ML, Deckers JW. Contribution of nursing to risk factor management as perceived by patients with established coronary heart disease. Eur J Cardiovasc Nurs. 2002;1(2):87-94.

7. Allen JK, Dennison-Himmelfarb CR, Szanton SL, Bone L, Hill MN, Levine DM, et al. Community outreach and cardiovascular health $(\mathrm{COACH})$ trial:a randomized, controlled trial of nurse practitioner/community health worker cardiovascular disease risk reduction in urban community health centres. Circ Cardiovasc Qual Outcomes. 2011;4(6):595-602

8. Wagner $\mathrm{EH}$. The role of patient care teams in chronic disease management. BMJ. 2000;320(7234):569-72.

9. Theobald K, McMurray A. coronary artery bypass grafting graft surgery: discharge planning for successful recovery. J Adv Nurs. 2004;47(9):483-91.

10. Zimmerman L, Barnason S, Nieveen J, Schmaderer M. Symptom management intervention in elderly coronary artery bypass grafting graft patients. Outcomes Manag. 2004;8(1):5-12.

11. Johnston G, Goss JR, Malmgren JA, Spertus JA. Health status and social risk correlates of extended length of stay following coronary artery bypass grafting. Ann Thorac Burg. 2004;77(2):557-62.

12. Zung WW. A self-rating depression scale. Arch Gen Psychiatry. 1965;12(1): 63-70.

13. Zung WW. A rating instrument for anxieties orders. Psychosomatics. 1971; 12(6):371-9

14. McGrath BM, Norris CM, Hardwicke-Brown E, Welsh RC, Bainey KR. Quality of life following coronary artery bypass grafting graft surgery vs.percutaneous coronary intervention in diabetics with multivessel disease: a five-year registry study. Eur Heart J Qual Care Clin Outcomes. 2017;3(3):216-23.

15. Park AH, Lee SJ, Oh SJ. The effects of a smoking cessation program me on health-promoting lifestyles and smoking cessation in smokers who had undergone percutaneous coronary intervention. Nurs Pract. 2015;21(2):10717.

16. Law MR, Wald NJ, Rudnicka AR. Quantifying effect of stations on low density lipoprotein cholesterol,ischemic heart disease,and stroke:systematic review and meta-analysis. BMJ. 2003;326(7404):1423.

17. Popejoy LL, Moylan K, Galambos C. A review of discharge planning research of older adults 1990-2008. West J Nurs Res. 2009;31(7):923-47.

18. van Walraven C, Oake N, Jennings A, Forster AJ. The association between continuity of care and outcomes:a systematic and critical review. J Eval Clin Pract. 2010;16(5):947-56

19. Price P. Physiologic effects of first-time sitting among male patients after coronary artery bypass grafting graft surgery. Dynamics. 2006;17(1):12-9.

20. Carroll A, Dowling M. Discharge planning communication education and patient participation. Br J Nurs. 2007;16(14):882-6.

21. Duits AA, Duivenvoorden HJ, Boeke S, Taams MA, Mochtar B, Krauss XH, et al. The course of anxiety and depression in patients undergoing coronary artery bypass grafting graft surgery. J Psychosom Res. 1998;45(2):127-38.

22. Gu G, Zhou Y, Zhang Y, Cui W. Increased prevalence of anxiety depression symptoms in patients with coronary artery disease before and after percutaneous coronary. BMC Psychiatry. 2016;16:259.

23. Martin F. Recognizing depression after a coronary artery bypass grafting graft. Br J Nurs. 2006;15(13):703-70.

\section{Publisher's Note}

Springer Nature remains neutral with regard to jurisdictional claims in published maps and institutional affiliations. 